\title{
Consumo De Marihuana Y Vulnerabilidad Del Derecho Al Libre Desarrollo
}

\section{Marijuana Use And Vulnerability To The Free Development Right}

\author{
(D) Diana Cabrera Becerra \\ (1) Daniel Cerna Álvarez \\ Rodrigo Uriel Velázquez Yépez ${ }^{3}$ \\ Judith Villafaña Benítez ${ }^{4}$
}

Recepción: Abril 15 de 2021 Aprobación: Mayo 22 de 2021 Publicación: Junio 30 de 2021

Cómo citar este artículo: Cabrera B, Diana. CernaÁ, Daniel. Velázquez Y, Rodrigo. Villafaña B, Judith (2021). "Consumo De Marihuana Y Vulnerabilidad Del Derecho Al Libre Desarrollo". Miradas, Vol. 16, No 1. pp. 46 - 58 https://doi.org/10.22517/25393812.24871

\section{Resumen}

Con miras a identificar las formas en que un consumidor puede ver violentado, se presentan los resultados de un grupo focal conformado por consumidores de marihuana. Se trabajó con una población joven y con acceso a fuentes de información y recursos electrónicos debido a las condiciones de aislamiento por la Covid 19 a nivel mundial. Se exploran y discuten las experiencias en donde se han visto violentados por

\footnotetext{
1 Egresada de Licenciatura en Psicología, Facultad de Psicología, UNAM; Programa Universitario de Derechos Humanos; UNAM; cabreradiana051@gmail.com

2 Lic. en Psicología, Facultad de Psicología, UNAM. Maestrante en Psicología Social, Universidad Autónoma Metropolitana, Plantel Iztapalapa; danceralv@gmail.com. ORCID: https://orcid.org/0000-0002-3506-5096

3 Egresado de Licenciatura en Psicología, Facultad de Psicología, UNAM; Programa Universitario de Derechos Humanos; UNAM; rodrigourielvelazquezyepez@gmail.com

4 Estudiante de Licenciatura en Psicología, Facultad de Psicología, UNAM; Programa Universitario de Derechos Humanos; UNAM; judithvibenitez@outlook.com
} 
el hecho de consumir, haciendo énfasis en la vulnerabilidad al derecho al libre desarrollo de su personalidad. Se destacan los comentarios en torno a las instituciones y figuras de autoridad, así como las estructuras sociales en donde interacción la interacción se ve afectada. En ese sentido, se concluye que los consumidores de marihuana con un grupo vulnerable que, además, ha sido invisibilizado a partir de los discursos institucionales de corte adultocentrista.

Palabras clave: Derecho al libre desarrollo; vulneración de derechos; consumidores; marihuana; juventud.

\section{Abstract}

In order to identify the ways in which a consumer may be victim of violence, the results of a focus group made up of marihuana consumers are presented. It was worked with a young population and with access to information sources and electronic resources due to the isolation conditions caused by Covid 19 worldwide. Experiences where they have been violated by the fact of consuming are explored and discussed, emphasizing the vulnerability to the right to free development of their personality. Comments about institutions and authority figures are highlighted, as well as social structures where interaction is affected. For this matter, it is concluded that marijuana users are a vulnerable group that, in addition, has been made invisible from the adult-centric institutional discourses.

Keywords: Free development right; violation of rights; consumers; marijuana; youth.

\section{Introducción}

No son pocos los estudios que se han hecho en torno al tema del consumo de drogas en adultos jóvenes. Dentro de la literatura al respecto, es común que el énfasis gire alrededor a las consecuencias que dicha actividad tiene sobre la salud, que van desde efectos crónicos, daños a ciertos órganos, aparición de enfermedades, entre otros efectos físicos y sociales, tales como el daño de las relaciones interpersonales, pérdida del trabajo, desintegración familiar, entre otros (Cáceres, et al., 2006; 522). De igual forma, es posible identificar el interés que existe respecto al origen o las causas que pueden detonar el consumo de drogas, desde aspectos cognitivos, como la percepción del riesgo hasta aspectos sociales, como el modelo de crianza (Becoña, 2007; 13,14). En este sentido, existe la viabilidad de inclusive hablar sobre el consumo precoz, cuando éste se da en sujetos de 11 años o menores, y las consecuencias que puede tener sobre ellos, como la dependencia (Hernández, et al., 2009; 201). Para dichos efectos, uno de los elementos que cobra mayor importancia es el núcleo familiar. Según Becoña, "cuando la familia proporciona adecuado apoyo social a sus hijos [...] es un predictor del no consumo de drogas de sus hijos (2007; 97).

Cabe señalar, el problema del consumo no es algo que se presente únicamente en la adolescencia o en etapas tempranas del desarrollo. También puede darse en el campo laboral; por mencionar un ejemplo, la gravedad de ello se puede apreciar en el hecho de que "según estudios de la 
International Labour Organization, los trabajadores que consumen alcohol y otras drogas presentan absentismo laboral de 2 a 3 veces mayor que el resto de los trabajadores" (Ochoa y Madoz, 2008; 27).

Sucede, sin embargo, que en dichos estudios e investigaciones, la posición con respecto al fenómeno de estudio, es decir, a la experiencia del consumo, es vista desde fuera, como si se siguiera el modelo que contrapone y divide a la sociedad en dos sectores: consumidores y no consumidores (Tsukame, 1999; 64).Si bien, recuperar una clasificación que data de 1999 podría parecer anticuado, en la inmediatez empírica se puede dar cuenta de su vigencia en la época actual. Sin embargo, al revisar datos más actuales, se observa que de 1991 a 2014, el consumo de drogas en jóvenes mexicanos aumentó de $8.2 \%$ a $17.2 \%$; a su vez, el consumo de marihuana aumentó del $1.5 \%$ al $10.6 \%$ (Villatoro, 2016; 201). Dichas cifras resultan alarmantes en términos cuantitativos, no obstante, lo que los números no muestran es la experiencia del consumidor. Si bien, hay un aumento en el consumo, es necesario considerar que la experiencia es de personas, no de números.

La problemática del consumo de drogas, entre sus consecuencias más graves, implica el reforzamiento de patrones de exclusión social, a raíz de los obstáculos para el desarrollo psicosocial, así como favorecimiento a la participación y aceptación social del individuo (Arriaga y Hopenhayn, $2000 ; 18)$. A esto refiere el fenómeno de vulnerabilidad social, donde se ubica la vulneración a los Derechos Humanos, el tema de principal interés para el presente trabajo.

No es posible ignorar el hecho de que "sociedades diferentes, con culturas diferentes, tienen distintas maneras de concebir la realidad, así como una misma sociedad transforma su manera de interpretar la realidad a consecuencia de su devenir histórico" (Slapak y Grigoravicius, 2007; 240); de ahí que sea necesario examinar la problemática del consumo de drogas desde un enfoque histórico, entendiendo cómo éste ha ido cambiando a lo largo del tiempo y, en primer lugar, cuestionarse si se trata, en efecto, de un problema.

De esta forma, vale la pena recordar que "pese a que el uso de drogas históricamente ha sido una conducta tolerada socialmente, en las sociedades modernas, sin embargo, su empleo ha tendido a ser restringido y monopolizado por las élites médicas" (Glavic y Barriga; 2005; 33). En esto, que puede conceptualizarse como sociedad, existe una objetivación del fenómeno de consumo de drogas, en donde se ve como algo negativo en cuanto a sus efectos sobre la salud. Sin embargo, es necesario recuperar el papel de la subjetividad del actor, del consumidor, que las muchas de las veces son vistos a la sombra de estos imperativos con respecto a la salud, olvidando sus razones, su propia experiencia y la forma en que puede ser violentado.

Las razones de dicha omisión pueden ser varias; una de ellas es que aun en pleno siglo XXI sigue presente el pensamiento racional positivista 
"que defiende el desarrollo, el progreso y la eficiencia, de tal suerte que todas las prácticas sociales (junto con sus usuarios) que vayan en otro sentido [...] pueden ser reprobadas, a decir de Ervin Goffman (1993)" (en Sánchez y Nateras, 2005; 65). Esto, aunado al contexto actual, marcado por sistema económico capitalista, donde la participación social se da con base en la aportación económica.

En este sentido, existe una estrecha relación entre la normatividad y la legalidad. Para ciertos sectores sociales, el consumo de drogas puede ser algo normal, en tanto, no deja de ser ilegal; la riqueza de ello consiste en el consumo como una práctica cotidiana, como elemento de la realidad del sujeto consumidor. Para algunos grupos sociales, por ejemplo, "el uso de la marihuana se convierte en una especie de artefacto o accesorio con valor simbólico [...], expresa determinada adscripción identitaria que conlleva un estilo y forma de ser y estar en el mundo" (Sánchez y Nateras, ídem). Así, sería arriesgado, a la vez que factible, considerar el consumo de drogas, en este caso, la marihuana, como una forma de expresión del ser del sujeto, como parte del derecho al libre desarrollo de la personalidad, es decir, "la capacidad natural que tienen todas las personas a decidir de manera libre sobre su desarrollo individual" (Senado de la República, 2018).

Se puede decir que el ejercicio de consumo de marihuana representa, más allá de la lid médica al respecto, una forma de ser, identificarse y ejercer los derechos del sujeto, en un mundo marcado por los imperativos morales con respecto a la salud y la estética. No obstante, dicha moralidad permea diferentes esferas sociales, instituciones inclusive; y, con ello, afecta al sujeto consumidor y le vulnera.

En vista de lo anterior, se refieren dos tipos de investigaciones con respecto al consumo de marihuana y otras sustancias: en primer lugar, aquellas que se interesan por el consumo per se, independientemente del actor o las condiciones en torno a las cuales ocurre; $y$, en segundo lugar, aquellas que dan cuenta de la existencia de un sujeto que, además de ser consumidor, es un ser humano y que, a su vez, no son ajenas a las condiciones políticas, sociales y económicas que atraviesan el fenómeno del consumo de sustancias.

El presente documento ubica sus intereses en el segundo tipo de trabajos. Para ello, es necesario replantearse epistemológicamente ante el fenómeno que se pretende estudiar. De tal suerte que resulta necesario problematizar la posición de enunciación con respecto al mismo. Si previamente se hablaba de élites médicas es porque el discurso desde la esfera de la salud no es otro que desde la esfera de poder. Así, es posible hablar de una historia del consumo de drogas y otras sustancias tóxicas, por ejemplo, la modernización de las institucione reguladoras de su consumo a partir de las ideas científicas de los imperios europeos desde el siglo XIX, (Enciso, 2015), o bien, en el caso de México, cómo las políticas de cuño conservador durante la supuesta reestructuración democrática del siglo XXI fueron restándole seriedad al tema del consumo (Pérez, 2016) y encauzaron los reflectores hacia el tema 
del combate al crimen organizado y el narcomenudeo, tema en el cual se puede apreciar, al menos, un elemento más: la juventud, ya que ésta es la población que se presenta como principal objetivo del mercado de drogas ilícitas (Conadic, 2011, en Zamudio, 2013; 112).

Por otro lado, es posible hablar de una contrahistoria al respecto del mismo fenómeno, entendida esta como "una forma de concebir la historia en contravía total con las formas tradicionales (Márquez, 2014; 214). Así, si existe una historia del fenómeno a partir de las instituciones, de corte conservador, prohibicionista y moralista, también es posible conocer una historia desde una posición diferente: la del consumidor. Para ello, resulta necesario replanetar la forma de pensarlo, pasando de la concepción clásica del sujeto cognoscible, es decir, que puede ser conocido, al sujeto cognoscente, bajo el planteamiento una epistemología del sujeto conocido (Vasilachis, 2006), la cual "reconoce al sujeto en su calidad de ser humano como interlocutor válido, en su esencia, y [que] puede aportar desde su perspectiva del mundo de la vida al concepto sobre el trabajo que se requiere en la investigación" (Cabrales, 2012; 73). Por otro lado, si el consumo está marcado por una moralidad eurocéntrica, como se ha mencionado, el problematizarlo se vuelve un ejercicio de descolonización del mismo, toda vez que esto supone, a su vez, una crítica al logos eurocéntrico (De Sousa, 2011; 17).

En todo caso, la primera posición que debe problematizarse es la de uno mismo como investigador, suponiendo que, como menciona Bourdieu, hemos de ubicar a quien escribe "como un actor, cuyo punto de vista depende de la posición que ocupa en el campo del que forma parte" (Giglia, 2002; 35). Así, la escritura no sólo es un ejercicio académico, sino político. En éste las voces pueden ser escuchadas, impuestas, omitidas o silenciadas. Bajo esta lógica de dominación, donde el discurso forma parte de una relación de poder, "la propensión a tomar la palabra es estrictamente proporcional al sentimiento de tener derecho a la palabra" (Bourdieu, 1998; 420). Si, como se ha discutido, el discurso con respecto al consumo parte de las instituciones, es porque éstas se han apropiado de él, presentándolo como el discurso oficial y tomando a los consumidores meras fuentes de información.

En el contexto de la pandemia por Covid 19 cabe señalar que los trabajos que se han llevado a cabo mantienen su énfasis en el acto del consumo y sus consecuencias, mas no en la experiencia del consumidor, pues trabajan desde un enfoque médico. Por ejemplo, se habla al respecto del peligro particularmente grave para quienes fuman marihuana, a raíz de cómo el Covid-19 ataca los pulmones (Volkow, 2020; 61), en el consumo como una forma de "aliviar el displacer" (Pascale, 2020; 333). Si bien, la postura médica con respecto a cualquier fenómeno cotidiano se reviste de gran importancia por el citado contexto, ésta no es la única forma de aproximación que existe.

Lo que se pretende con este trabajo es analizar el fenómeno del consumo desde la propia experiencia 
de sus actores y no a partir de la institucionalización del mismo, con relación a la vulnerabilidad de sus derechos que hayan podido llegar a vivir.

\section{Metodología}

Para llevar a cabo dicha tarea, y en aras de evitar el uso de estadísticas y datos numéricos, se optó por el empleo de grupo focales toda vez que éstos permiten "captar el sentir, pensar y vivir de los individuos, provocando auto explicaciones para obtener datos cualitativos" (Hamui y Varela, 2012; 56). Otra forma de conceptualizarlos es como "conversaciones colectivas o entrevistas grupales" (Kamberelis y Dimitriadis, 2005; 494). La riqueza conceptual que pueden aportar es vasta, ya que no sólo se incentiva la participación, sino el diálogo y la discusión en un espacio seguro, respetuoso y moderado por miembros del propio equipo de investigación.

En la presente investigación se llevó a cabo un estudio cualitativo trabajando con un grupo focal conformado por jóvenes consumidores, con una duración de dos horas, integrado por jóvenes tanto mujeres como hombres. Como apunte crucial es necesario indicar que, debido a las condiciones actuales que se viven por la pandemia de Covid-19, esta técnica fue llevada a cabo mediante una plataforma digital en tiempo real.

Participaron seis adultos jóvenes, cuatro hombres y dos mujeres, habitantes de la zona metropolitana de México. Debido a complicaciones derivadas del uso de las herramientas digitales, como la pérdida de conexión a internet $\mathrm{o}$ del sonido o video, fueron tres integrantes del propio equipo quienes moderaron la sesión, siguiendo la estructura de introducción, establecimiento de afinidad y profundización del tema, así como cierre y conclusiones, a partir de una guía de preguntas que giran en torno a cómo han sentido vulnerados sus derechos a partir de su experiencia de consumo.

El desarrollo del grupo se llevó a cabo de la siguiente manera: comenzamos con la mención del consentimiento informado, así como una explicación de la dinámica a seguir durante la sesión. Seguido de ello, una breve presentación de los participantes, donde el nombre era opcional. Al respecto, un factor representativo del grupo fue la edad, ya que todos los participantes eran menores a 27 años, de tal forma que pueden ser considerados jóvenes y adultos jóvenes.

Cabe señalar que el uso de cámara web fue una decisión que cada uno de ellos pudo tomar libremente. Se trabajó con una guía conformada por nueve preguntas, de las cuales las primeras dos fueron meramente introductorias conforme a los intereses y motivos de los participantes; la última pregunta sirvió para cerrar la sesión y dar pie a las conclusiones; en tanto, las preguntas de la tres a la ocho refieren a los cuestionamientos medulares para el tema de la vulnerabilidad a sus derechos por el hecho de ser consumidores de alguna sustancia; entre éstas, la pregunta ocho incluye un enfoque de género derivado de los intereses actuales en la materia 
Se siguió la estructura del modelo recuperado del trabajo de Acosta y Gonzáles-Celis (2009). El contenido de las preguntas es el siguiente:

1: ¿Cómo han visto afectadas sus actividades a partir de que consumen?

2: ¿Qué opinan sobre la información que se da con respecto a las drogas?

3: ¿Te has sentido discriminado por el hecho de ser consumidor?

4: ¿Cuáles han sido los espacios donde has sufrido discriminación?

5: ¿Has intentado tomar algún tratamiento o intervención para dejarlo?

6: ¿Qué dificultades han experimentado al ser consumidores? 7: ¿Cómo te ha afectado el hecho de ser mujer-consumidora?

8: ¿Bajo qué estereotipos has vivido a raíz de ser consumidor?

9: ¿Qué opinas del consumo de estupefacientes?

Para cerrar, se destinó un espacio para comentarios finales y conclusiones.

\section{Resultados}

Con base en lo anterior, se obtuvieron una serie de respuestas que muestran heterogeneidad en cuanto a la experiencia del consumo, ya que cada persona es un actor diferente, con su propio habitus (Bourdieu, 2000, op. Cit), que no son ajeno a las condiciones sociales bajo las que vive. De ahí la necesidad de recuperar estos elementos, ya que "las inclinaciones (habitus) son inseparables de las estructuras que las producen y las reproducen" (íbid; 33).

Apartirdeesteejercicio,pudimos recuperar los siguientes fragmentos de las respuestas de los participantes del grupo focal, que se presenten a partir de la pregunta correspondiente, según lo más significativo en ellas.

\section{1: ¿Cómo han visto afectadas sus actividades a partir de que consumen?}

* "Llevo consumiendo 8 años marihuana [...]. De repente fumo demasiado $\mathrm{y}$ a veces creo que sí podría entorpecer algunas actividades".

* "En el día a día me distraigo un poco, hace que deje de hacer ciertas actividades".

* "Fumar me ha enseñado a administrarme, en dinero, en tiempo, en muchas cosas".

* "Si tengo algo que hacer, si, si tengo clases lo regulo, lo administro, lo uso para relajarme".

\section{2: ¿Qué opinan al respecto de las personas de la calle que consumen drogas?}

* "Yo tengo acceso a una educación de calidad, a muchos factores. Pero, ¿qué hay, por ejemplo, de los niños de la calle?"

* "Me sentaba a platicar y encontraba historias muy interesantes; además ellos se sentían muy bien por el hecho de que alguien los tomara en cuenta, de que alguien los escuchara".

* "Es muy, muy raro que alguien se siente a escuchar ese tipo de personas".

* "A quien consume, somos nosotros mismos quienes los estamos alejando; quienes vamos caminando por la calle y los miramos con ojos extraños, no los miramos como miramos a alguien con traje". 
3: ¿Te has sentido discriminado por el hecho de ser consumidor?

* "No he tenido malos tratos, pero sí un alejamiento de algunas personas". * "Yo sentí un acosototal. Yuna forma de verme como menospreciada".

\section{4: ¿Cuáles han sido los espacios} donde has sufrido discriminación?

* "Alguna vez tuve un problema con un doctor. Fui a una consulta y le comente que había consumido drogas un día antes y que yo creía que a eso se debía mi malestar, entonces esta persona comenzó a tratarme diferente"

* "Yo he sufrido discriminación en lugares que se me hace una estupidez que la haya, por ejemplo la escuela". * "Si tienes algún accidente y vas marihuano, te dejan como hasta el último. La banda drogadicta en el hospital no importa. Esa ausencia misma está impartida por la sociedad, obligan a alguien a ser ausente".

* "Quienes consumimos no estamos eximidos de la delincuencia. Levantar un acta, la denuncia, acudir al Ministerio Público, es un proceso tratado, muy burocrático; y si le sumas el hecho de consumir, es otra limitante en el acceso a la justicia".

\section{5: ¿Has intentado tomar algún} tratamiento o intervención para dejarlo?

* "No he buscado ayuda profesional. No he sentido que la necesito pero sí he querido disminuir mi consumo".

* "Nunca he pasado por un tratamiento. Yo creo en apelar que sea como una práctica disruptiva de la vida, pues podría ser más problemática, yo creo que cuando tomas un tratamiento es de antemano ponerte del lado del enfermo"

* "Yo sí. Note que ya era demasiado y busqué reducirlo porque sentí que no estaba poniendo atención a lo que aprendía o se me olvidaba muy rápido, como que las cosas se me olvidaban muy rápido en general". * "Yo no he tomado tratamientos; considero que es por esta misma cuestión de verte como paciente, de verte como si tuvieras algo mal. Yo nunca me he visto como paciente en este aspecto".

\section{6: ¿Qué dificultades han experimentado al ser consumidores?}

* "Habría que enfatizar en la cuestión de salud e higiene del consumo; ir a consumir cualquier cosa implica cierto tipo de productos o sustancias que vienen adheridas, así como cuando fumas un cigarro que tiene miles de sustancias químicas adicionales".

" "Estar conflictuado por las situaciones en casa, por lo que la gente opine de ti. Habría que posicionarnos, no en el aspecto de que caemos en la adicción, sino habría que asumir la adicción, como una cuestión fuera de nosotros" * "A lo mejor el conflicto ni siquiera es ser marihuano es ser juzgado por los que quieres"

* "He ido a ciertos lugares y me arrepiento muchísimo. He estado en situaciones desagradables, terribles, me he salvado de varias".

* "Tengo que seguir yendo al baño a fumar mis toques, porque es algo 
que no, aun no se comprende y no se acepta".

* "En un parpadeo de ojos pues yo estaba en el mundo de las drogas, no? sin querer y más cuando eres chiquito pues a quien le dices? ¿A quién le pides ayuda? si tus papas te dicen que está mal, a lo mejor tú no lo quisiste pero ahora ya que estas ahí, ¿qué? ¿Quién te ayuda?".

* "Una señora me comentaba que estaba sentada en el camellón, y unos perros se pelearon al lado, se paró, se fue para un local; se acostó enfrente y se durmió; salió el dueño del local y le echó agua fría".

\section{7: ¿Cómo te ha afectado el hecho} de ser mujer-consumidora?

* "El hecho de ser mujer me pone en un riesgo más. Siempre tengo que depender de mi hermano, de un amigo, alguien que decirle ¿oye tú por favor puedes ir?, y tratar de comprarlo. Para mí es más difícil consumirla".

* "Uno se expone al ir a los puntos. Es muy complicado, sobre todo para una que es mujer, porque, generalmente los dealers son hombres. Nunca falta el que te coquetea, o cosas así"

\section{8: ¿Bajo qué estereotipos has} vivido a raíz de ser consumidor?

* "Si eres marihuano eres como cholo y ratero"

* "A lo mejor ya ni quieres ser adicto, ni consumir. Me pasó que me decían: “¿Siempre estás pacheco? Les decía "no, nada más estoy pacheco para aguantar estas preguntas" De porqué te tengo que andar dando una explicación de algo".
9: ¿Qué opinas del consumo de estupefacientes?

" "Siento que ya eso de las adicciones ya es meramente pues político y social".

* "La marihuana para mí es mucho menos mala que el alcohol, que el cigarro, y se tiene una peor connotación".

* "Yo creo que es mucho más sana la marihuana que el alcohol y el cigarro; no te".

* Hay gente que consume cantidades exorbitantes de pornografía, de internet, de chocolate, de cafeína; que realmente son demasiado dañinos y se siguen promoviendo; porque lo que impera en el mercado pues es simplemente el consumo".

* "Yo soy fiel acreedor de que habría que salirse de ese aspecto peyorativo que representa el consumir algún tipo de droga".

* "Siento que la mota une; es ese espíritu de hermandad".

* "Estas adicciones se dan por el rechazo social".

* "Nunca en mi vida he visto a alguien que fume marihuana y se ponga agresivo; al contrario, nos ponemos un poco más amigables". * "Me parece injusto la forma en la que tengo que adquirirla. Me gustaría comprarla en un lugar decente como otras personas compran otras sustancias, en ese sentido, es lo que he sentido: cierta opresión, una injusticia".

\section{Comentarios finales}

* "Logré superar algunos obstáculos que yo tenía, y todo eso sin dejar de fumar. Hasta entré a la universidad". 
* "Yo creo que este estigma de que es ilegal, y creo que quienes más nos juzgan son personas adultas".

* "Yo creo que sería más factible, quizás, vislumbrar la pérdida del derecho. Y desde ahí tratar de exigir si quieren una clase de inclusión, pero no a partir de lo patológico".

\section{Discusión}

A partir de las respuestas compartidas por los participantes, es posible dar cuenta de ciertas situaciones que los afectan en cuanto al derecho al libre desarrollo de la personalidad. Tomando en cuenta que la primera mención al respecto de este derecho data del 23 de mayo 1949, en la Ley Fundamental de la República Federal de Alemania, en su artículo 2.1 (Villalobos; 2012; 63), el camino a trazar en México es aún largo, siendo que apenas en el siglo XXI es cuando este concepto recobra de relevancia social.

Se recuperan tres características del derecho al libre desarrollo de la personalidad a partir del trabajo de Johan Villalobos (2012), a saber: a) que "busca proteger y tutelar los diversos aspectos indispensables de la dignidad y calidad de persona humana" (2012; $65)$; b) "que la persona humana goce efectivamente de todo el sistema de libertades y derechos fundamentales" (2012; 66); y c) "tutelar el desarrollo particular de cada individuo (ídem).

Para cada una de estas, existen respuestas de los participantes en donde la vulneración a dicho derecho se ha manifestado de diversas índoles, ubicando la situación en México, donde los derechos fundamentales pueden dividirse en cuatro grandes rubros, a saber: principios generales, derechos y libertades personales, derechos sociales, económicos y culturales y principios rectores de las actividades de los poderes públicos (Carbonell, 2001; 182).

En este sentido, se presentan dos derechos fundamentales de todo ser humano. En primera, el derecho a la salud, del cual la OMS (Organización Mundial de la Salud) nos señala, "significa que todo el mundo debe tener acceso a los servicios de salud que necesita [...] sin ser objeto de violencia o discriminación (Adhanom, 2017). También hacemos referencia al derecho al acceso a la justicia, "el derecho de toda persona a tener un ámbito en el cual hacer valer el derecho de que se crea asistida y de lograr la satisfacción de ésta (Marabotto; 2003; 291).

Para ambos casos, las respuestas de los participantes muestran cómo se han dado situaciones que vulneran dichos derechos e, inclusive, en alguna ocasión se habla sobre acoso por parte del personal médico.

$\mathrm{Si}$ recuperamos las tres características que plantea Villalobos, daremos cuenta, a partir de las respuestas de los participantes, que su libre derecho a la personalidad ha sido vulnerado de diversas formas: desde la omisión de un trato digno en diferentes espacios, desde el público, como las calles, hasta el privado, como en su propio hogar; cabe señalar que la violencia se da de forma estructural desde las instituciones, como las de salud o jurídicas y hasta la familia misma. 
Para el caso de la segunda característica, como se habló renglones más arriba, los consumidores han sido violentados en cuanto al goce de sus derechos fundamentales; se hace mención, inclusive, de la falta de información para sectores de la población menos privilegiados: las personas que viven en la calle, los jóvenes sin acceso a la información y la infoxicación que existe al respecto.

De esta forma, la tercera característica se ve vulnerada en cuanto, a raíz del consumo, las personas ponen en riesgo su integridad, ya sea al acudir a los puntos, o lugares en donde se compra dicho producto, así como a los estereotipos bajo los que viven e, inclusive, el rechazo social del cual son objeto.

\section{Conclusiones}

Las consecuencias de todo lo anterior son vastas. Como se puede observar, la falta de información y aceptación al respecto provoca que, en la omisión del tema, el consumidor se muestre incapaz de pedir ayuda, en caso de considerarlo necesario. Se pone énfasis, a su vez, en el papel del prohibicionismo. Si bien, el debate en México sigue presente, lo que muestran las respuestas de los participantes es que, independientemente de su legalización, el consumo provoca distanciamiento social. Sin embargo, la dinámica se torna diferente cuando vemos que los propios consumidores guardan una dinámica propia; de unión, como señala un participante.

En este sentido, la dinámica del grupo de consumidores sigue una lógica propia, diferente, por ejemplo, a quien los ve desde fuera del grupo. Se señala que los juicios provienen, en su mayoría, de la población adulta. El tema del adultocentrismo no es ajeno al de la vulnerabilidad de su derechos. Se insiste que, en ocasiones, dicha dinámica se puede apreciar desde el núcleo familiar.

Existe, a su vez, un sentido de injusticia con respecto al consumo. Al parecer, el prohibicionismo sobre las drogas, en este caso, la marihuana, obedece a intereses políticos y no, necesariamente, de salud pública. Para quien consume, el problema no gira en torno a lo patológico, sino a la inclusión. Se puede dar cuenta de ello a partir de los juicios que se llevan a cabo con respecto a la regulación de otras sustancias, como el alcohol, y las consecuencias que tiene sobre la salud y la convivencia con el otro. Como señalan, existe una peor connotación con respecto al consumo de marihuana, aunque ésta, por ejemplo, no te provoque resaca tras su consumo, o bien, como señalan en otra participación, en el caso de la agresividad que pueden presentar quienes consumen otro tipo de sustancias, frente a lo amigable que puede ser quien consume marihuana.

A su vez, cabe señalar que, pese a la dinámica social de segregación a partir del consumo, ésta no es ajena a cuestiones de clase ni de género. La forma en que pueden agraviar a los sujetos es diferente cuando se trata de personas de clase media-alta, como los participantes de la técnica del estudio, a personas que viven en situación de calle, como ellos mismos narran. De igual forma, los riesgos a los que las mujeres consumidoras se 
enfrentan son diferentes a los de los hombres consumidores. Lo grave de esta situación es que seguirán siendo vulnerables ante tales situaciones en tanto el acceso a la misma siga siendo prohibitivo.

Por último, se menciona que, pese a los prejuicios sociales que existen con respecto a los consumidores y de los cuales ellos son conscientes, éste no es, necesariamente, un limitante para el cumplimiento de sus metas. En ese sentido, la resiliencia parece factor clave para el desarrollo libre de la personalidad, en un contexto lleno de violencia y discriminación.

\section{Referencia bibliográfica}

Acosta, C. y González-Celis, A. (2009). Actividades de la vida diaria en adultos mayores: la experiencia de dos grupos focales. Revista Psicología y Salud, 19 (2), pp. 393-401.

Adhanom, T. (2017). La salud es un derecho humano fundamental (10 de diciembre de 2017). Recuperado de: https://www.who. int/mediacentre/news/statements/ fundamental-human-right/es/\#. YDGir7ytfiY

Arriagada, I., \& Hopenhayn, M. (2000). Producción, tráfico y consumo de drogas en América Latina. CEPAL.

Becoña, E.. (2007). Bases psicológicas de la prevención del consumo de drogas. Papeles del psicólogo, 28(1) pp. 11-20.

Becoña, E. (2007). Resiliencia y consumo de drogas: una revisión. Adicciones, 19 (1), pp. 89-101.

Bourdieu, P. (2000). La dominación masculina. Anagrama
Cabrales, O. (2012). La epistemología del sujeto conocido y la concepción del trabajo en Colombia. Itinerario educativo, (59), pp. 53-78.

Cáceres, D., et al. (2006). Consumo de drogas en jóvenes universitarios y su relación de riesgo y protección con los factores psicosociales. Universitas Psychologica, 5 (3), pp. 521-534.

Carbonell, Miguel. (2001). Los derechos fundamentales en la Constitución mexicana: una propuesta de reforma. Isonomía, (14), pp. 181193.

De Sousa, B. (2011). Epistemologías del sur. Utopía y Praxis Latinoamericana, 16 (54), pp. 17-39.

Enciso, F. (2015). Nuestra historia narcótica: Pasajes para (re) legalizar las drogas en México. Debate.

Giglia, A. (2002). Para comprender a Bourdieu. Sobre su teoría y práctica de la entrevista. Trayectorias, 4 (10), pp. 27-40.

Glavic M. \& Barriga O. (2005). Hacia una comprensión del consumo de marihuana. Ciencias Sociales Online, 2 (1), pp. 31-44.

Hamui-Sutton, A., \& Varela-Ruiz, M. (2013). La técnica de grupos focales. Investigación en educación médica, 2(5), pp. 55-60.

Hernández, T. et al. (2009). La Edad de Inicio en el Consumo de Drogas, un Indicador de Consumo Problemático. Psychosocial Intervention, 18(3), pp. 199-212.

Kamberelis, G., \& Dimitriadis, G. (2005). Focus Groups: Strategic Articulations of Pedagogy, Politics, and Inquiry. In N. K. Denzin \& Y. S. Lincoln (Eds.), The Sage handbook of qualitative research. Sage Publications Ltd. 
Marabotto, J. (2003). Un derecho humano esencial: el acceso a la justicia. Anuario de Derecho Constitucional Latinoamericano, pp. 291-301.

Márquez, J. (2014). Michael Foucault y la Contra-Historia. Historia $y$ memoria, (8), pp. 211-243.

Ochoa E., \& Madoz A. (2008). Consumo de alcohol y otras drogas en el medio laboral. Medicina y seguridad del trabajo, 54(213), pp. 25-32.

Pascale A. Consumo de sustancias psicoactivas durante la pandemia por COVID-19. Rev. Méd. Urug. 36(3), pp. 333-334.

Pérez, R. (2016). Tolerancia y prohibición. Aproximaciones a la historia social y cultural de las drogas en México (1840 - 1940). DEBATE.

Sánchez A. \& Nateras A. (2005). Los contextos del uso de marihuana en Latinoamérica y culturas juveniles urbanas en México. Revista Española de Drogodependencias, 30 (1-2), pp. 50-71.

Senado de la República (2008). Derecho a la autodeterminación y al libre desarrollo de la personalidad, urgen en el Senado. Categoría: Boletines (02 de octubre de 2018). Recuperado de: http:// comunicacion.senado.gob. mx/index.php/informacion/ boletines/41950-derecho-a-laautodeterminacion-y-al-libredesarrollo-de-la-personalidadurgen-en-el-senado.html

Slapak, S., \& Grigoravicius, M. (2007). "CONSUMO DE DROGAS": LA CONSTRUCCIÓN DE UN PROBLEMA SOCIAL. Anuario de investigaciones, 14, 239-249.

Tsukame, A. (1999). El consumo de drogas en busca de sentido. Revista de la Academia, (4), pp. 63-75.
Vasilachis, I. (2006). Estrategias de investigación cualitativa. Vol. I. Gedisa Editorial.

Villalobos, K. (2012). El desarrollo humano al libre desarrollo de la personalidad. Universidad de Costa Rica. Facultad de Derecho.

Villatoro, J., et al. (2016). El consumo de drogas en estudiantes de México: tendencias y magnitud del problema. Salud mental, 39(4), pp. 193-203.

Volkow, D. (2020). Collision of the COVID-19 and addiction epidemics. Ann Intern Med, 173 (1), pp. 61-62.

Zamudio, A. (2013). Jóvenes en el narcomenudeo: el caso de la Ciudad de México. URVIO: Revista Latinoamericana de Estudios de Seguridad, (13), pp. 111-123. 